\title{
Querying Semistructured Data with Compression in Distributed Environments
}

\author{
B.M. Monjurul Alom, Frans Henskens, Michael Hannaford \\ School Of Electrical Engineering and Computer Science, University Of Newcastle, \\ Callaghan, NSW 2308, Australia
}

\begin{abstract}
As data management applications grow more complex, they are likely to need efficient distributed query processing. In Distributed Database Systems complete replication consists of maintaining complete copies of the database at each site; this has advantages such as highest locality of reference, highest reliability, availability, and is best for reading. The most promising and dominant data format for data processing and representing on the Internet is the semistructured data form termed XML. XML data has no fixed schema; it evolved and is self describing which results in management difficulties compared to, for example relational data. It is therefore a major challenge for the database community to design query languages and storage methods that can retrieve semistructured data. In this paper, we present a storing and querying scheme for semistructured data views of relational form in distributed environments. The proposed technique stores path dictionary, word dictionary, attribute dictionary, and the complete compressed replication of semistructured data in each distributed site of the DDBS. The presented technique provides query performance improvement due to the compression of semistructured data.
\end{abstract}

Key Words: Bitmap Indexing, Distributed database, Dictionary, XML, XQuery.

\section{Introduction}

Distributed data management has been an important domain of research since the early days of databases [1]. Distributed DBMSs reflect organizational structure, improving shareability, local autonomy, availability, reliability, performance, and economy of use. By distributed database, we mean a logically interrelated collection of shared data (and a description of this data), physically distributed over a computer network [2]. With the development of the Web and the existence of universal standards for data exchange, the problem of data management arguably became the most challenging to the database community [3]. With the heterogeneity and autonomy of sources, it is now even more difficult. Generally, structured or relational data can be stored in distributed sites without too much difficulty, whilst storing semistructured data in distributed sites requires appropriate techniques so that the stored data can be retrieved in an efficient way without loss of information.

Semistructured data is becoming more and more prevalent for use in performing simple integration of data from multiple sources [4]. By semistructured, we mean that although the data may have some structure, this structure is not as rigid, regular, or complete as the structure required by traditional database management systems [4]. The emergence of XML [5], which is a data format for semi-structured data, has increased the use of semistructured data, assisted by the fact that attribute names are stored with the data itself, making it selfdescribing [6].

XML is the dominant data exchange format for Internet-based business applications. It is also used as the data format for automated tasks such as information extraction, natural language processing, and data mining [7]. When in XML form, data is neither table-oriented as in a relational database, nor is it strictly typed as in an object database [4]. Rather, XML data comprises hierarchies that have no fixed schema. While XML form supports Internet transport and certain data processing tasks, it causes issues for other common activities such as querying and updating.

One option for managing semistructured as well as XML data is to build a specialized data manager that contains a XML data repository at its core [8]. It is difficult to achieve high query performance using XML data repositories, since queries are answered by traversing many individual element to element paths requiring multiple index lookups. In the case of XML data, queries are even more complex because they may contain regular path expressions [9]. Additional flexibility is needed in order to traverse data whose structure is irregular or partially unknown to the user. Techniques exist for querying XML data such as Lorel[10], XML-QL[11] , XQL[12], XPath[13], XQuery[14], UnQL[15], XML-GL[12], XSL[12], Quilt[16]; however these query languages are complicated to use and have some limitations.

XQL [12] uses a tree structured model of the $\mathrm{XML}$ data. It does not support nested queries, so no new elements can be added to an existing query. In XQL there is no clause that specifies the order of the result. In 
XML-QL there is no range selection neither does this query language support query reduction, negation nor support audio, video, images or specialized data types such as JPEG. Lorel [10] also does not support query reduction but does supports audio, video, images and specialized data types such as JPEG, and uses the object exchange data model. XPath is mainly understood as a language for selecting a subset of the nodes of an XML document tree but all major XPath engines take exponential time in the size of the input query [17]. XQuery is a more standard, powerful query language, easy and flexible to use, also supporting ordered, group by, aggregate functions [16]. But XQuery sometimes leads to unexpected query results which prevent index exploitation[18]. Query rewrite and optimization is more complex for XML queries than relational queries [18].

Another option for managing semistructured data is to store and query it with a relational database [8]. In the database community many researchers argue that the relational (and object-relational) model still is the best option due to its maturity and widespread usage [16]. The data must be converted into a set of tuples and stored in tables. This process requires a schema for the data. Relational databases are traditionally queried based on the value of some attributes [9]. The problem of efficient query evaluation on distributed, semistructured data is described in [19]. In this approach the nodes of the database are distributed over a fixed number of sites and the edges are classified into local edges (with both ends at the same site) and cross edges (with ends in two distinct sites).

This paper describes a technique for storing and querying of semistructured data with compression. The technique, termed BIQS (Bitmap Indexing and Querying Semistructured data) represents XML data in a compressed form that complies with relational structure, supporting the use of relational tools such query languages for data manipulation. The technique has been designed to be suitable for distributed storage of XML data to create distributed XML databases. The technique uses word, element, and attribute dictionaries and a compressed semistructured database structure in each of the distributed sites. The word dictionary stores all the distinct words and corresponding tokens for each word, from all the XML documents. An element path dictionary is used to record all the distinct element paths (epaths) and their path number. An attribute dictionary is used to store all the attribute names, their values, and associated document numbers.

We also support a dynamically created database structure to record the element paths, contents of the element paths, XML attributes, and all the document numbers in which words, element paths, and attributes exist. This structure is compressed without losing any $\mathrm{XML}$ information and consequently, data retrieval can be performed efficiently with low latency. The proposed technique stores and queries semistructured data in a more space and time efficient way than is provided by other existing schemes. We have compared our presented searching technique with other query techniques such as XQuery, and SQL in Oracle to evaluate the searching efficiency.

The remainder of this paper is organized as follows: the structure of the proposed method is described in section 2. Querying semistructured data (database) is presented in section 3. Section 4 presents experimental results and analysis of storage capacity. The paper concludes with a discussion and final remarks in section 5 .

\section{Structure of the Proposed Technique}

The overall structure of the proposed technique is presented in Figure 1. The technique generates a word dictionary, a path dictionary, and an attribute dictionary, which together become the basis of a compressed database structure to store XML document information. The compressed database structure and all the dictionaries are stored in each site of the distributed database system, as shown in Figure 2. End-users can query the semistructured data from each of the distributed sites.

The presented technique dynamically creates a database structure that represents the existence of all the words, element paths and XML attributes in the corresponding documents. The first row of the database structure records all the token numbers for the corresponding words, XML attributes and the associated path numbers for the words and attributes. All the tokens and XML attributes exist (are bounded) within their corresponding path number in the first row of the structure.

Each XML attribute is stored against its respective token number, in order to support retrieval of exact XML information. Specially, the XML attributes that could be used as a relational primary key are stored next to each token. We use a negative (-) sign before all path numbers to distinguish from tokens or attributes. The first column of the structure stores the document number. The entries of the database structure use a bit value (1/0) to represent the existence or not of the word, element path, or attribute within the document.

To get a new path from an XML document, the approach initially generates a new column in the DB structure. The first row (entry) of the column stores the path number (from the path dictionary) and a value 1 is inserted to the next row of the created column. The value 1 represents the path's existence in the document. The tokens (from the word dictionary) of all the words within the selected path number are stored similarly by creating new columns in the DB structure. The values of XML attributes are also stored in columns after each token 
column within the selected path number, in the structure. A value 1 is inserted into the next entry of each of the created columns for the token and attribute. Each row of the structure records all the information of each distinct XML document. Similarly the system completes the database structure creation for all XML documents.

This system does not create new columns, within an existing path, for the same word and same attribute even for different documents. It creates new columns (for words and attributes) within an existing path, for the same word but different attributes, even for different documents. The technique always creates new columns for same the word but different path number, whatever the attributes and document number. In the following discussion, consider the XML documents given in Figure 3, Figure 4, Figure 5, and Figure 6 for BIQS database structure construction.

The word dictionary, path dictionary and attribute dictionary (comprising Tables 1, 2 and 3) have been created from the XML documents shown in Figures 3 to 6. The system creates a new column in the database structure (given in Table 4) to get the path name "FamilyInfo.Personal.Fname" from the document ${ }_{1}$ (given in Fig. 3), the path number (-1) is assigned to the first row of the created column, and a value 1 is assigned to the next row of the created column for the existence of the document 1 . For all the words within this path number, a new separated column is created and a value 1 is assigned to the next row of the created column for their existence in the corresponding document. Thus the token 1 and 6 for the words Turner and Duke are recorded within the path number 1 . The values of the XML attribute SSN are 5, 9 also recorded in the next column of each token $(1,6)$ of the words Turner and Duke. The value 1 is assigned to the next row of the created column for their existence in the corresponding document. In Table- 4,1 and 6 are two tokens, 5 and 9 are two attributes, and -1 is the path number 1 .

The database structure after extracting all the words, paths and attributes from the document 1 , is given in Table 5. It continues to build this structure until all the XML documents have been searched. The final structure, presented in Table 6, represents all the words and element paths from the XML documents shown in Figures 3 to 6 .

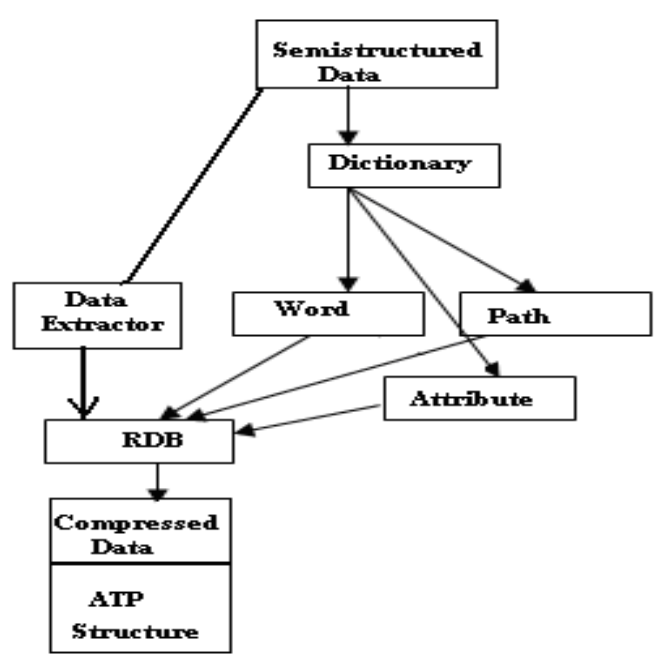

Figure 1. Structure of the BIQS.

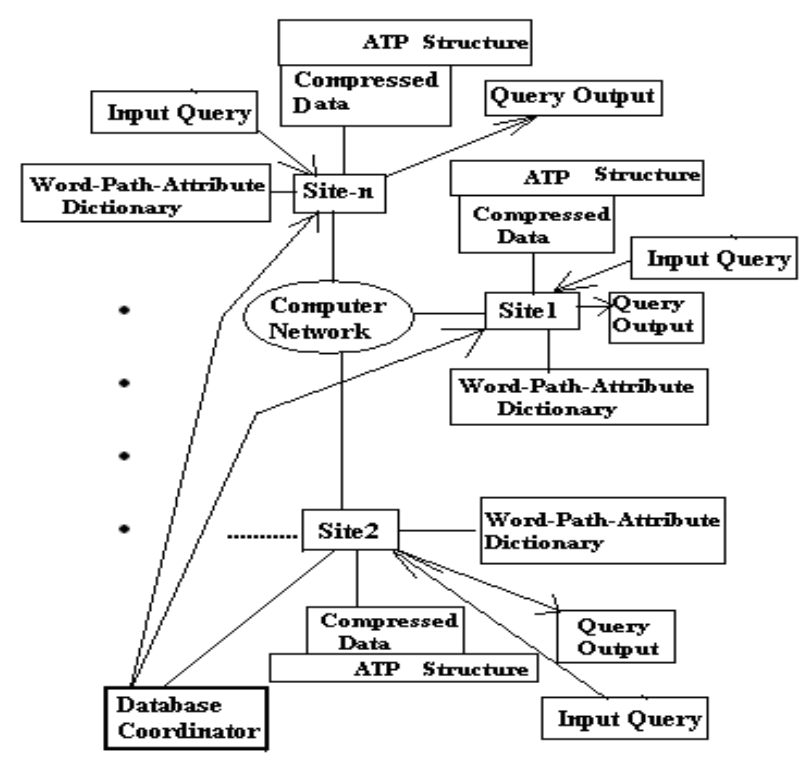

Figure 2. Compressed semistructured data in distributed environment.

The system separates the database structure into two different structures to compress the XML data. The first row is named the Attribute Token and Path (ATP) structure and is used to represent the token, path number and attributes. This row is indexed serially starting from 0 . These indexes are used later for searching the token and path number from the Token and Path structure. Another structure named Compressed Data Structure consists of all other remaining rows of the complete database structure (except the ATP row). Each row of the database structure is divided into blocks. In each block, the information is compressed using 16 bit cells, and because each row represents the information from each XML document, there would typically be a different 
number of blocks for each document, and each block consists of different values for different documents.

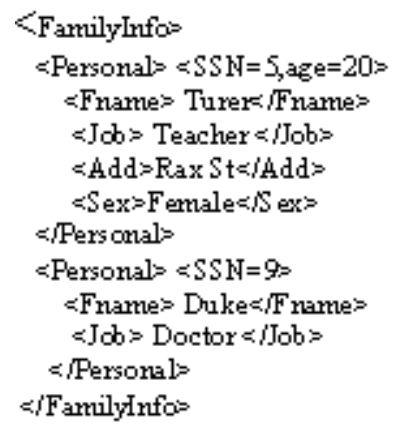

Figure 3. Document,

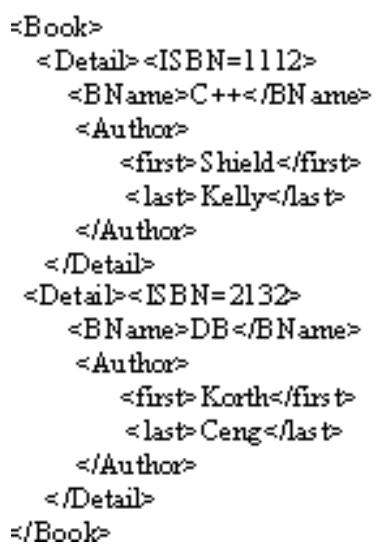

Figure 5. Document ${ }_{3}$.

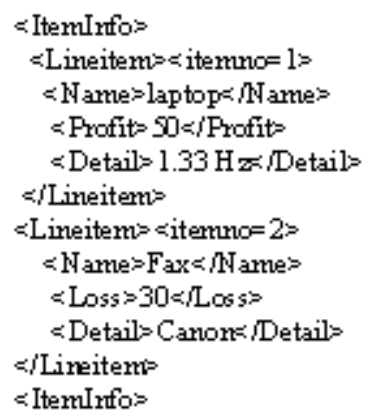

Figure 4. Document ${ }_{2}$.

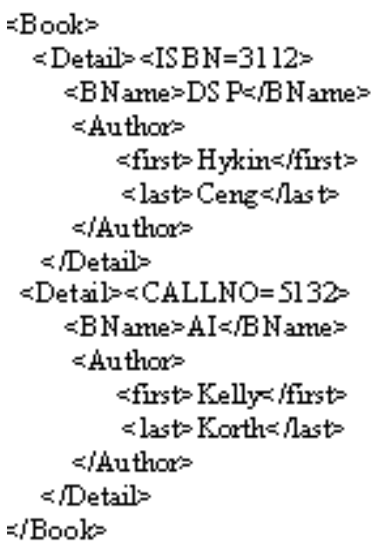

Figure 6. Document

Compression is also possible using 32 bit cells. The ATP structure is presented in Table 7, and the compressed bitmap structure is presented in Table 8; the value of each 16 bit cell is shown in decimal form. If there is insufficient data to form a block with 16 bit cells, we fill these bits with zero. The compressed data structure is shown in Table 8, the first column of the structure represents the document number and the other four columns represent the blocks. The value of each block is generated from the database structure given in Table 6. The values of the $\mathrm{Block}_{0}$ are 65535, 0, 0 and 0 . These values represent the compressed form of data for different XML documents. This compression does not lose any information. We use the compressed data structure for searching the semistructured data.

\section{Querying the Database}

The BIQS technique supports different types of querying and searching, applied to the compressed data structure. The ATP structure is stored in each site, and is used to obtain the index of the token and attributes within a path boundary.
Table 1. Word Dictionary.

\begin{tabular}{|l|l|}
\hline Token & Vahx \\
\hline 1 & Thmer \\
\hline 2 & Teacher \\
\hline 3 & Rax \\
\hline 4 & St \\
\hline 5 & Female \\
\hline 6 & Duke \\
\hline 7 & Doctor \\
\hline 8 & Laptop \\
\hline 9 & S0 \\
\hline 10 & 1.33 \\
\hline 11 & Hx \\
\hline 12 & Fax \\
\hline 13 & 30 \\
\hline 14 & Canon \\
\hline 15 & C++ \\
\hline 16 & Shì $\mathbf{H}$ \\
\hline 17 & Kelk \\
\hline 18 & DB \\
\hline 19 & Korth \\
\hline 20 & Cerg \\
\hline 21 & DSP \\
\hline 22 & Hyim \\
\hline 23 & AI \\
\hline & \\
\hline
\end{tabular}

Table 2. Path Dictionary.

\begin{tabular}{|c|c|}
\hline Pro & Pathriame \\
\hline 1 & Familyifo. Personal. Fhame \\
\hline 2 & Familyrfo. Personal Job \\
\hline 3 & Familyhro.Personal.Add \\
\hline 4 & Familififo.Personal.Sex \\
\hline 5 & Itembrfo. Lineitem Hame \\
\hline 6 & Itemfrfo.LineitemProfit \\
\hline 7 & Iteminfo.Lineitem Detail \\
\hline 8 & Iteminfo.Line item Loss \\
\hline 9 & Book.DetsilBNarme \\
\hline 10 & Book.DetsildAuthor $\mathrm{finst}$ \\
\hline 11 & Book.Detsilfurthor.last \\
\hline
\end{tabular}

Table 3. Attribute Dictionary.

\begin{tabular}{|ll|l|}
\hline Arame & Vahue & Docomertro \\
\hline SSH & 5 & 1 \\
\hline SSH & 9 & 1 \\
\hline age & 20 & 1 \\
\hline temmo & 1 & 2 \\
\hline temno & 2 & 2 \\
\hline ISBH & 1112 & 3 \\
\hline ISBH & 2132 & 3 \\
\hline ISBH & 3112 & 4 \\
\hline CALLHO & 5132 & 4 \\
\hline
\end{tabular}

Each distributed site also stores the word, path and attribute dictionaries. Users search the word dictionary to find the token for the corresponding word; after obtaining the token, the system finds the index position of the token from the Token and Path structure, and the path number in which the token is bounded. Because information for 16 memory cells is compressed in each block, the Block no is calculated, dividing the index number by 16 . The Offset position is also calculated as 
the index number modulo 16. From the compressed data structure (Table 8), the corresponding values of each block are converted into binary forms to check the word's existence in the document.

This approach checks the existence of value 1 in the corresponding offset position in each of the block values. The presence of the value 1 in the corresponding offset position indicates the existence of the word in the corresponding document number. The system can search for a single word or for multiple words. To search for an element-path (and path contents), the system initially searches the path number within the path dictionary and then searches all the token numbers within this path number from the ATP structure. After retrieving the token number, the corresponding words are searched for in the word dictionary. If searching for an attribute (from an XML document), the technique can search directly from the attribute dictionary.

Table 4. Structure after completing Path-1.

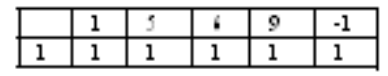

Table 5. Structure after extracting all data from document-1.

\begin{tabular}{|l|l|l|l|l|l|l|l|l|l|l|l|l|l|l|l|l|l|l|}
\hline & 1 & 5 & 6 & 9 & -1 & 2 & 5 & 7 & 9 & -2 & 3 & 5 & 4 & 5 & -3 & 5 & 5 & -4 \\
\hline 1 & 1 & 1 & 1 & 1 & 1 & 1 & 1 & 1 & 1 & 1 & 1 & 1 & 1 & 1 & 1 & 1 & 1 & 1 \\
\hline
\end{tabular}

Table 6. Structure of the data completing all the paths (from all documents).

\begin{tabular}{|c|c|c|c|c|c|c|c|c|c|c|c|c|c|c|c|c|c|c|c|c|c|c|c|c|c|c|c|c|}
\hline & 1 & 5 & 6 & 9 & -1 & 2 & 5 & 7 & 9 & -2 & 3 & 3 & 4 & 5 & -3 & 3 & 5 & -4 & $\$$ & 1 & 12 & 2 & -5 & 9 & 1 & -6 & 10 & 1 \\
\hline 1 & 1 & 1 & 1 & 1 & 1 & 1 & 1 & 1 & 1 & 1 & 1 & 1 & 1 & 1 & 1 & 1 & 1 & 1 & 0 & 0 & 0 & 0 & 0 & 0 & 0 & 0 & 0 & 0 \\
\hline 2 & 0 & 0 & 0 & 0 & 0 & 0 & 0 & 0 & 0 & 0 & 0 & 0 & 0 & 0 & 0 & 0 & 0 & 0 & 1 & 1 & 1 & 1 & 1 & 1 & 1 & 1 & 1 & 1 \\
\hline 3 & 0 & 0 & 0 & 0 & 0 & 0 & 0 & 0 & 0 & 0 & 0 & 0 & 0 & 0 & 0 & 0 & 0 & 0 & 0 & 0 & 0 & 0 & 0 & 0 & 0 & 0 & 0 & 0 \\
\hline 4 & 0 & 0 & 0 & 0 & 0 & 0 & 0 & 0 & 0 & 0 & 0 & 0 & 0 & 0 & 0 & 0 & 0 & 0 & 0 & 0 & 0 & 0 & 0 & 0 & 0 & 0 & 0 & 0 \\
\hline
\end{tabular}

\begin{tabular}{|l|l|l|l|l|l|l|l|l|l|l|l|l|l|l|l|l|l|l|l|l|l|l|l|l|l|l|}
\hline & 11 & 1 & 14 & 2 & -7 & 13 & 2 & $-S$ & 15 & 1112 & $1 S$ & 2132 & 21 & 3112 & 23 & 5132 & -9 & 16 & 1112 & 19 & 2132 \\
\hline 1 & 0 & 0 & 0 & 0 & 0 & 0 & 0 & 0 & 0 & 0 & 0 & 0 & 0 & 0 & 0 & 0 & 0 & 0 & 0 & 0 & 0 \\
\hline 2 & 1 & 1 & 1 & 1 & 1 & 1 & 1 & 1 & 0 & 0 & 0 & 0 & 0 & 0 & 0 & 0 & 0 & 0 & 0 & 0 & 0 \\
\hline 3 & 0 & 0 & 0 & 0 & 0 & 0 & 0 & 0 & 1 & 1 & 1 & 1 & 0 & 0 & 0 & 0 & 1 & 1 & 1 & 1 & 1 \\
\hline 4 & 0 & 0 & 0 & 0 & 0 & 0 & 0 & 0 & 0 & 0 & 0 & 0 & 1 & 1 & 1 & 1 & 1 & 0 & 0 & 0 & 0 \\
\hline
\end{tabular}

\begin{tabular}{|l|l|ll|ll|ll|ll|lll|ll|l|}
\hline & 22 & 3112 & 17 & 132 & -10 & 17 & 1112 & 20 & 2132 & 20 & 3112 & 10 & 512 & -11 \\
\hline 1 & 0 & 0 & 0 & 0 & 0 & 0 & 0 & 0 & 0 & 0 & 0 & 0 & 0 & 0 \\
\hline 2 & 0 & 0 & 0 & 0 & 0 & 0 & 0 & 0 & 0 & 0 & 0 & 0 & 0 & 0 \\
\hline 3 & 0 & 0 & 0 & 0 & 1 & 1 & 1 & 1 & 1 & 0 & 0 & 0 & 0 & 1 \\
\hline+ & 1 & 1 & 1 & 1 & 1 & 0 & 0 & 0 & 0 & 1 & 1 & 1 & 1 & 1 \\
\hline
\end{tabular}

Table 7. ATP structure.

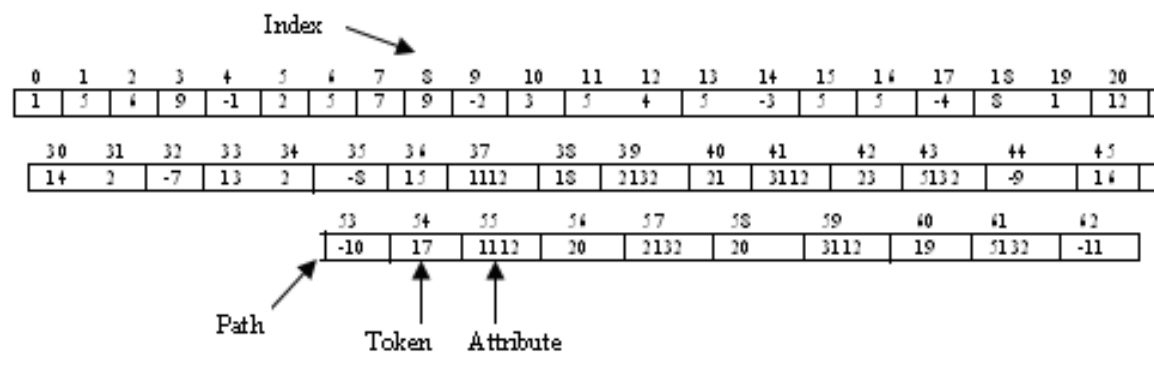

Table 8: Structure of compressed data.

\begin{tabular}{|c|c|c|c|c|}
\multicolumn{1}{c}{ Doc No } & \multicolumn{1}{c}{ Block $_{0}$} & \multicolumn{1}{c}{ Block $_{1}$} & \multicolumn{1}{c}{ Block $_{2}$} & \multicolumn{1}{c}{ Block $_{3}$} \\
\hline 1 & 65535 & 49152 & 0 & 0 \\
\hline 2 & 0 & 16383 & 61440 & 0 \\
\hline 3 & 0 & 0 & 3855 & 34754 \\
\hline 4 & 0 & 0 & 248 & 31806 \\
\hline
\end{tabular}

The functionality of the searching scheme is demonstrated in the following examples:

Query \#1: Find all the information about author Korth from all XML documents (Shown in Figs. 3 to 6).

The query language as follows can be applied to any of the distributed sites:
Select * From documents Where author= "Korth".

The technique searches for the token of the word "Korth" and it is 19 from the word dictionary. This token exists within path numbers 10 and 11 (from the ATP structure). The system finds the index of the token (from token and path structure), that is 47 (within path number 10), and 
60 (within path number 11). The method calculates Block_no $=47 / 16=2$; and offset_position $=47 \% 16=15$. The compressed information (from compressed data structure) is compressed into binary form from Block_no $=2$. The stored values in $B_{1}$ lock $_{2}$ are 0, 61440, 3855 , and 248. The binary values are 0000000000000000, 1111000000000000 , 0000111100001111, and 0000000011111000. The offset position (15) is found to have a value 1 for the word existing in the corresponding document. We see only the $3^{\text {rd }}$ value ( $3^{\text {rd }}$ row in Block-2) 0001111000011111 has a 1 in $15^{\text {th }}$ position (starting from left to right, 0 to 15). So the word "Korth" is present in document 3 with the path number 10 named Book.Detail.Author.first. But Korth is not present in document $_{1}$, document or document $_{4}$, (as a first author i.e.with path number 10).

Similarly for index number 60 this word is found within path number 11 that is "Book.Detail.Author.last". The system searches the word in Block_no 3 (Block_no= $60 / 16=3$, offset position $=60 \% 16=12$ ). The values of Block no 3 are searched for the existence of 1 in their offset position 12, and is found in Document 4 . So Korth is found as a first name (path number 10) in document 3 and as a last name (path number 11) in document 4 . We compare this result to the original XML documents shown in Figs. 3 to 6 and see it is correct.

Query \# 2: Find all the Fname (First name) whose Job is Doctor. The query language can be applied to any of the distributed sites as follows:

Select Fname From documents

Where Job= "Doctor".

The system supports these types of query (considering the existence of an XML attribute which is a primary key). The search technique searches for the token of the word Doctor and finds it to be 7 from the word dictionary. This token exists within path number 2 and the token number is also followed by the value of attribute 9, from the ATP structure. The index of token 7 is 7 from the token and path structure. So the Block no $=7 / 16=0$ and the offset position $=7 \% 16=7$. The system searches only the values of Block no 0 , from the Compressed data structure (Table 8) and the values are $65535,0,0,0$. The binary forms of these values are $1111111111111111,0,0,0$. The first value $\left(1^{\text {st }}\right.$ row of the Block-0) 1111111111111111 has a 1 in its offset position 7. So Doctor is present in document ${ }_{1}$ whereas it is not present in documents 2,3 , and 4 .

To find Fname, the technique finds the path number of Fname (it is part of FamilyInfo.Personal.Fname) from path dictionary and its path number is 1 . The token is searched for within the path number 1 and the values 1 and 6 (from path and token structure) are found. But token 1 is followed by the XML attribute 5 whereas token 6 is followed by the XML attribute 9. We also see the Token number of word "Doctor" is 7 which is followed by the XML attribute 9. Hence the system selects only the token 6 as both tokens 6 and 7 are followed by the XML attributes 9. In this way the XML attribute is used as a relational primary key to find the exact information. The word for the token 6 is found from word dictionary to be Duke. We see from document $_{1}$, only the Fname (First Name) Duke has Job listed as "Doctor".

\section{Experimental Results}

We used Oracle 9i (Enterprise Edition Release 9.2.0.8.0) and Stylus Studio 2008 XML Enterprise Suite Release 2 , to evaluate the different query results in the case of a centralized database system. We also used the $\mathrm{C}++$ language using BorlandC compiler (32 bit that supports up to 4 GB RAM) to implement our proposed technique. We used an Intel Processor with $2.13 \mathrm{GHz}$, 1.99 GB of RAM under the Windows XP professional operating system.

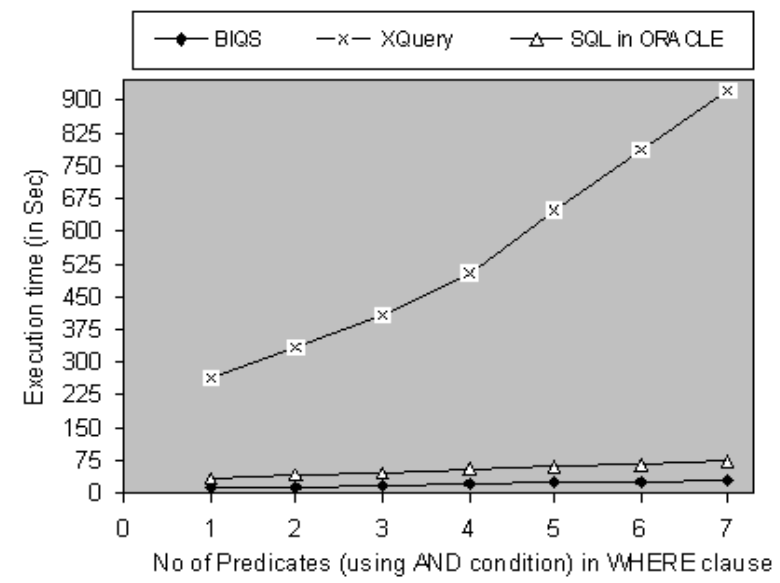

Figure 7. Query execution time analysis.

To support the Oracle 9i database we used the Linux operating system. We used the (well structured) XML datasets in [20] to run comparisons of the XQuery language and our proposed BIQS technique using file sizes of $1.0 \mathrm{MB}, 6.5 \mathrm{MB}, 13 \mathrm{MB}, 26 \mathrm{MB}$ and $130 \mathrm{MB}$. The experimental results for XQuery execution time, execution time in Oracle and BIQS are presented in Figure 7, which for the purpose of clarity only depicts the results for $130 \mathrm{MB}$ files, but which show comparative performance that is typical of all the tested file sizes.

It can be seen that BIQS execution times were substantially lower than those of XQuery, and also that BIQS outperforms the highly regarded Oracle execution times across the range of predicates tested. The results show that the improvement achieved by BIQS increases with the number of predicates.

A second set of experiments was performed to compare the query execution times of BIQS and Bitcube[21]. These experiments used 5, 10, 15, 20 and 25 element 
paths (ePaths) per document, and a variety of words per element path. For all numbers of ePaths per document, and all numbers of documents, BIQS outperformed. Bitcube as shown in Figure 8, which for the purpose of clarity only depicts the results for 25 ePaths per document, and 20 words per ePath.

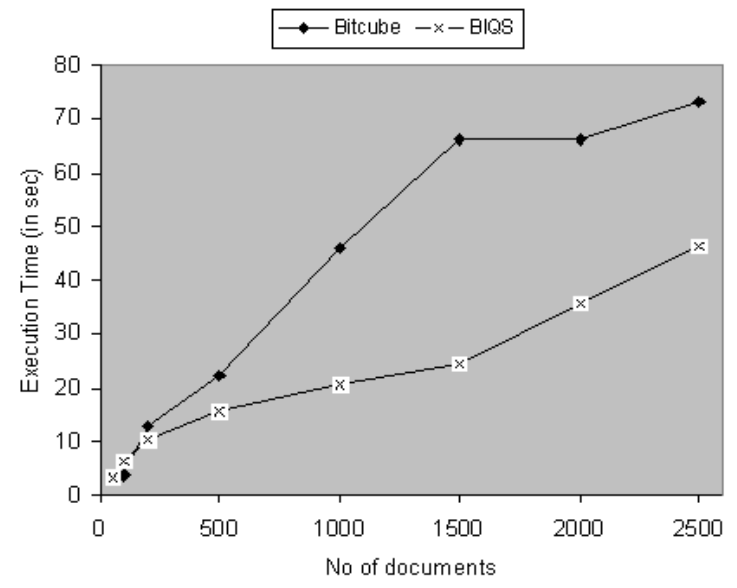

Figure 8. Execution time wrt word/ePath.

\section{Conclusion and Future work}

A (DDBMS) distributed database management system provides facilities such as local autonomy, no reliance on a central site, continuous operation, location independence, fragmentation independence, distributed query processing and distributed transaction processing. In this paper we present BIQS (Bitmap Indexing and Querying Semistructured Data), a new (semistructured) database structure incorporating data compression, which stores information about words, paths, and attributes of XML documents. Each of the distributed sites stores a replica of the whole database structure (both compressed data and attribute token and path (ATP)) and all the dictionaries (word, element, attribute).

We implemented the proposed BIQS technique using a centralized database system, and measured performance across a range of data sizes and properties. The query execution time of BIQS was found to be substantially better than Xquery [14] query language. This improvement was achieved through compression of the semistructured data to create a relational view, allowing use of the more efficient relational query languages. Although BIQS takes some initial time to construct the dictionary, after this it is very efficient at query evaluation. The execution time for query operations of BIQS was slightly better than Oracle (SQL query language), with the performance improvement increasing with the number of predicates. The execution time of BIQS also demonstrates superior time efficiency when compared to the highly regarded Bitcube [21] technique.
Issues such as database updating, recompressing, and optimal query execution in a distributed environment will be reported as the research progresses.

\section{References}

[1] P. Valduriez and T. Ozsu, "Principle of Distributed Database Systems.," Prentice Hall, 1999.

[9] T. Milo and D. Suciu, "Index Structures for Path Expressions," in ICDT Jarujalem, Israel, 1999.

[10] S. Abiteboul, D. Quass, J. McHugh, J. Widom, and J. L. Wiener, "The Lorel Query Language for Semistructured Data," International Journal on Digital Libraries, vol. 1(1), pp. 68-88, 1997.

[11] A. Deutsch, M. Fernandez, D. Florescu, A. Levy, and D. Suciu, "XML-QL: A Query Language for XML," http://www.w3.org/TR/NOTE-xml-ql.

[12] A. Bonifati and S. Ceri, "Comparative Analysis of Five XML Query Language," SIGMOD, vol. 29:1, pp. 68-79, 2000.

[13] M. Benedikt, W. Fan, and F. Geerts, "XPath Satisfiability in the Presence of DTDs," in PODS Baltimore, Maryland, 2005.

[14] S. Pal, I. Cseri, O. Seeliger, M. Rys, G. Schaller, W. Yu, D. Tomic, A. Baras, B. Berg, D. Churin, and E. Kogan, "XQuery Implementation in Relational Database System," in The 31st International Conference on Very Large Databases Trondheim, Norway, 2005.

[15] P. Buneman, M. Fernandez, and D. Suciu, "UnQL: a query language and algebra for semistructured data based on structural recursion," The VLDB Journal, vol. 9, pp. 76-110, 2000.

[16] A. A. d. Sousa, J. L. Perira, and J. A. Carvalho, "Querying XML Databases," in The 12th International Conference of the Chilean Computer Science Society (SCCC) IEEE, 2002. 
[17] G. Gottlob, C. Koch, and R. Pichler, "The Complexity of XPath Query Evaluation," in PODS San Diego, CA, 2003.

[18] A. Balmin, K. S. Beyer, and F. Ozcan, "On the Path to Efficient XML Queries," in The 32nd International Conference on Very Large Databases(VLDB) Seoul, Korea, 2006.

[19] D. Suciu, "Distributed Query Evaluation on Semistructured Data," ACM Tranascation on Database Systems, vol. 27:1, pp. 1-62, 2002.

[20] http://www.cs.washington.edu/research/xmldatasets/.

[21] J. P. Yoon, V. Raghavan, and V. Chakilam, "BitCube: A Three-Dimensional Bitmap Indexing for XML Documents," Journal of Intelligent Information Systems, vol. 17, pp. 241-254, 2001. 